\title{
Belgeo
}

Revue belge de géographie

4 | 2018

Transformations of urban space after the fall of

Socialism

\section{La fragmentation de l'espace urbain postsocialiste en Albanie}

\section{Alain Jarne}

\section{CpenEdition}

\section{Journals}

Édition électronique

URL : http://journals.openedition.org/belgeo/30849

DOI : $10.4000 /$ belgeo.30849

ISSN : 2294-9135

Éditeur :

National Committee of Geography of Belgium, Société Royale Belge de Géographie

Référence électronique

Alain Jarne, "La fragmentation de l'espace urbain postsocialiste en Albanie », Belgeo [En ligne], 4 ]

2018, mis en ligne le 08 janvier 2019, consulté le 10 décembre 2020. URL : http://

journals.openedition.org/belgeo/30849; DOI : https://doi.org/10.4000/belgeo.30849

Ce document a été généré automatiquement le 10 décembre 2020.

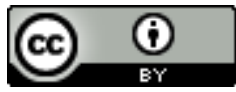

Belgeo est mis à disposition selon les termes de la licence Creative Commons Attribution 4.0 International. 


\title{
La fragmentation de l'espace urbain postsocialiste en Albanie
}

\author{
Alain Jarne
}

1 En quoi l'approche des espaces urbains albanais en tant que « postsocialistes » peut-elle s'avérer féconde pour saisir par contraste, d'une part, avec la période du développement socialiste, d'autre part, avec d'autres espaces urbains contemporains, les aspects spécifiques de leur devenir? L'Albanie ne constitue en un sens pas un terrain privilégié pour ce type d'approche : son histoire, à maints égards si singulière, a produit des espaces urbains qui eux aussi se distinguent davantage d'un hypothétique modèle de ville postsocialiste qu'ils ne s'en approchent. Tosic (2003) par exemple, déclinant huit types de villes postsocialistes, distingue les villes albanaises dans une catégorie à part, « proches du développement sauvage (unregulated) des villes du TiersMonde». Mais d'un autre côté la très grande force de la doctrine et la volonté d'exemplarité dans l'établissement du réseau des villes albanaises durant le communisme - cette « image spatiale de l'édification du socialisme» (Jandot, 1981) s'est peut-être prolongée après 1991 dans les villes les plus «postsocialistes» qui soient, parce que situées dans le seul État européen qui, après sa rupture avec l'URSS au début des années 1960, se prévaut d'avoir continué de toutes ses forces à travailler à l'« édification intégrale de la société socialiste " (Jandot, 1981). Ce constat nous ramène à la distinction entre la « ville postsocialiste » conçue comme modèle urbain, distinguée et décrite comme telle (Hirt, 2013), et cette même ville abordée sous l'angle de la trajectoire historique, c'est-à-dire des continuités/discontinuités sociales et institutionnelles entre le socialisme et le régime qui lui fait suite. Dans le second cas, la ville est abordée sous l'angle des traductions spatiales des mécanismes socioéconomiques et politiques de la transition: suburbanisation, gentrification et ségrégation au niveau intra-urbain, ainsi qu'une intégration progressive dans l'économie globale trouvant son expression spatiale dans la métropolisation (Coudroy de Lille, 2016). A l'instar de l'expérience socialiste, la transition albanaise a été d'une grande intensité, marquée en particulier par les importantes migrations (émigration et migrations internes vers les villes) et la métropolisation rapide. 
2 Dans les années 1970, la question posée par les théoriciens de la ville socialiste portait sur la réelle spécificité de celle-ci versus un simple différentiel des rythmes de développement dans un processus caractérisé essentiellement et partout au $20^{\mathrm{e}}$ siècle par l'industrialisation et la modernisation, qu'elles soient les produits du socialisme ou de l'économie de marché. Hamilton et French (1979) ont été au cœur de l'école dite historique (Hirt, 2013) ${ }^{1}$ pour laquelle une économie politique et des modes de production différents ont déterminé des formes et des fonctions urbaines sensiblement différentes. Si on adopte cette posture, on peut relayer certaines spécificités observables propres aux villes dites socialistes (Szelenyi, 1996, cité par Hirt, 2013): faible urbanisation (faible part de population urbaine au niveau national), faible urbanité (moins de mixité fonctionnelle et de diversité sociale) et structure spatiale spécifique (générosité des espaces publics, importants blocs résidentiels standardisés, conglomérats industriels, etc.). La transition se décline en processus (décentralisation, développement $\mathrm{du}$ commerce et des services, restitutions foncières et immobilières, désindustrialisation, polarisation sociale) qui travaillent les formes urbaines héritées, produisant le plus souvent suburbanisation et métropolisation, mais aussi une augmentation de la mixité ainsi que certaines formes de ségrégation (comme la gentrification) que les États socialistes avaient souvent su contenir sans toutefois les annuler (Hamilton et French, 1979 ; Coudroy de Lille, 2011). Nous cherchons ici à tracer deux propriétés prêtées aux État socialistes, à savoir le faible niveau d'urbanisation et un certain déficit d'urbanité des villes, par-delà la transition albanaise. L'accent est mis sur la question de la mobilité résidentielle et des migrations qui, encouragées aprèsguerre (Daniel, 1989), strictement contrôlées dès les années 1960 (Sjöberg, 1994) et brutalement libérées dans les années 90, déterminent des rythmes d'urbanisation séquencés, des formes urbaines contrastées et la réapparition d'une ségrégation spatiale présente avant-guerre et aux débuts du communisme. Nous commençons par la brève histoire d'une urbanisation exceptionnellement faible jusqu'en 1991 et, en dépit de la forte émigration, en rapide augmentation depuis.

\section{Histoire d'une sous-urbanisation}

On peut considérer pour ce qui concerne l'urbanisation de l'Albanie moderne cinq grandes périodes :

4 1. la stagnation de l'entre-deux guerres, à l'exception de Tirana qui se développe comme centralité en tant que capitale du nouvel Etat albanais ;

2. le décollage plus général mais tardif de l'urbanisation (comme chez les voisins balkaniques mais avec plus de 20 ans de retard sur l'URSS) dans les 15 années d'aprèsguerre, une période de croissance urbaine typique du premier stade de développement des pays socialistes, liée donc à l'industrialisation massive ${ }^{2}$ avec, dans la continuité de la période précédente, des niveaux particulièrement élevés à Tirana ainsi que dans les villes de la plaine centrale (Elbasan, Durrës, Fier et Vlorë) ;

3. une période courant de 1960 à 1991 marquée par la création de villes nouvelles et une stricte limitation de la croissance des villes existantes;

4. la reprise de l'urbanisation à la chute du communisme, grâce à l'apport de populations rurales venant s'installer dans de nouvelles banlieues «informelles » et autoconstruites ;

5. à partir du milieu des années 2000 , la formation de grands ensembles le plus souvent 
suburbains et pour la plupart construits dans et autour de Tirana (« aire métropolitaine Tirana-Durrës »), alors que les autres villes commencent à décliner.

5 La véritable singularité albanaise dans le contexte de l'Europe de l'Est est le virage important pris en 1960 suite au changement de la politique extérieure : placée dès lors dans l'orbite de la Chine maoïste, l'Albanie enclenche sa "révolution culturelle", marquée entre autres par un ensemble de politiques anti-urbaines, avec la volonté d'abolir les rapports de domination ville-campagne, ce qui se traduit en particulier par le maintien d'un équilibre numérique entre les deux. L'Albanie prend ses distances d'avec le modèle soviétique de l'accumulation socialiste primitive au profit d'une stratégie de "croissance nulle " où l'on cherche à accumuler le capital dans les communes rurales et à y reconvertir le surplus agricole dans le développement d'une industrie locale (Murray, Szelenyi, 1988). Dans un contexte d'isolement international et d'un pouvoir autoritaire imprimant une grande homogénéité idéologique, l'Albanie démontre sa capacité d'appliquer de manière forte et cohérente les principes de l'économie planifiée (Aliaj, 2003). Si les taux de croissance urbaine demeurent importants, ils sont désormais liés à une natalité générale très forte ; seules les petites villes nouvelles continuent de bénéficier d'un certain apport migratoire. Ce réseau de villes « exemplaire » s'effondre progressivement après 1991, essentiellement au profit de l'aire métropolitaine Tirana-Durrës. Si certaines villes résistent dans un premier temps - les départs vers Tirana ou l'étranger étant compensés par un apport régional de population rurale - toutes perdent depuis les années 2000 de la population (à l'exception de Saranda, cité balnéaire, seule ville à croître hors de la zone métropolitaine).

Figure 1. Evolution de la part de la population urbaine en (ex-)URSS, en Albanie et en Chine. Sources : compilation de diverses sources (avant 1960), Banque Mondiale (dès 1960).

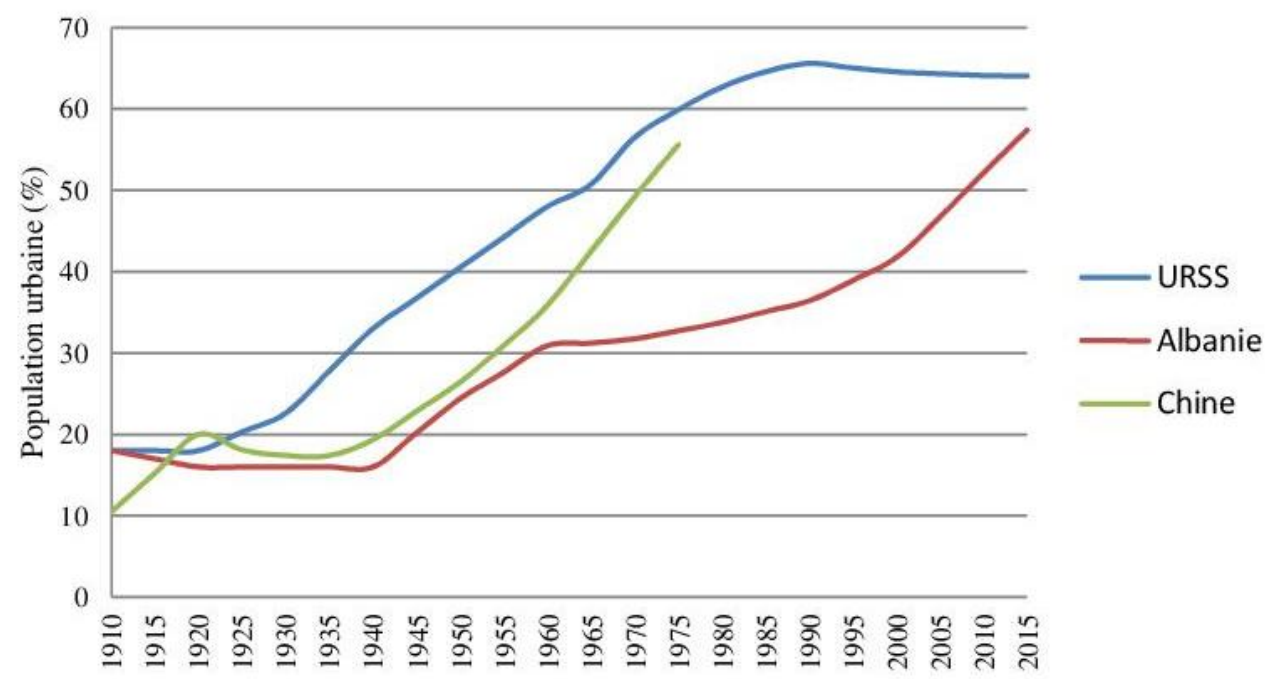

6 Après le démarrage industriel classique (le «Grand bond en avant» des Chinois) échelonné pour les trois régions, la Chine connaît une véritable désurbanisation lors de la Grande famine et de la Révolution culturelle, suivie en cela dans une mesure moindre par l'Albanie d'Enver Hoxha. Ce phénomène est unique en Europe de l'Est. A la chute du Mur, le niveau d'urbanisation de l'Albanie avoisine les $36 \%$, dépassé même par les 
moins développées des républiques ex-yougoslaves qui, dans l'ensemble, gagnent 20 points de pourcent entre 1960 et 1990, contre 6 pour l'Albanie (de 30 à $36 \%$ ).

Figures 2 et 3 . Urbanisation de l'Albanie, par types de villes.

\section{Population en valeurs absolues}

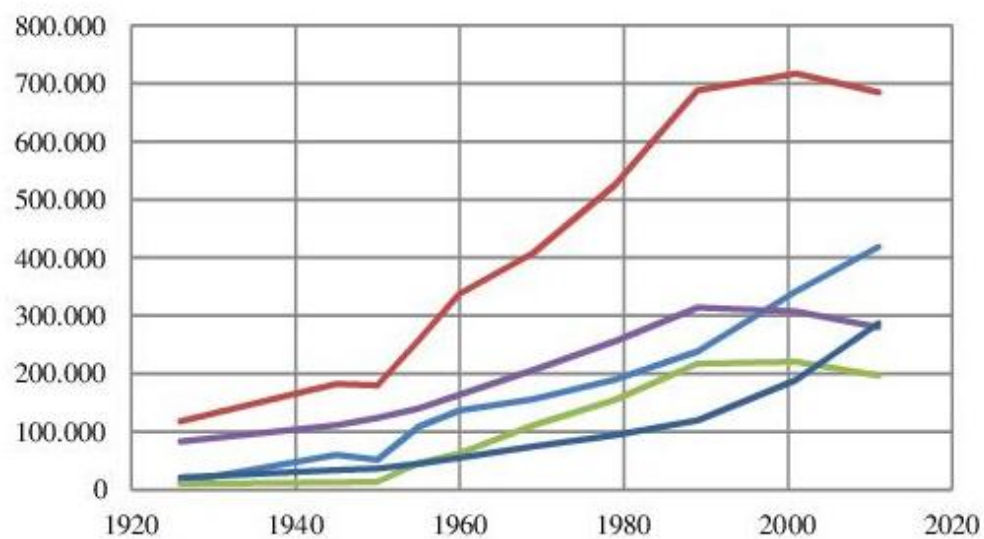

- Tirana

—Villes pré-communistes

Villes nouvelles

- Albanie (population/10)

- Suburbain Tirana

\section{Part de l'urbain par types de villes}

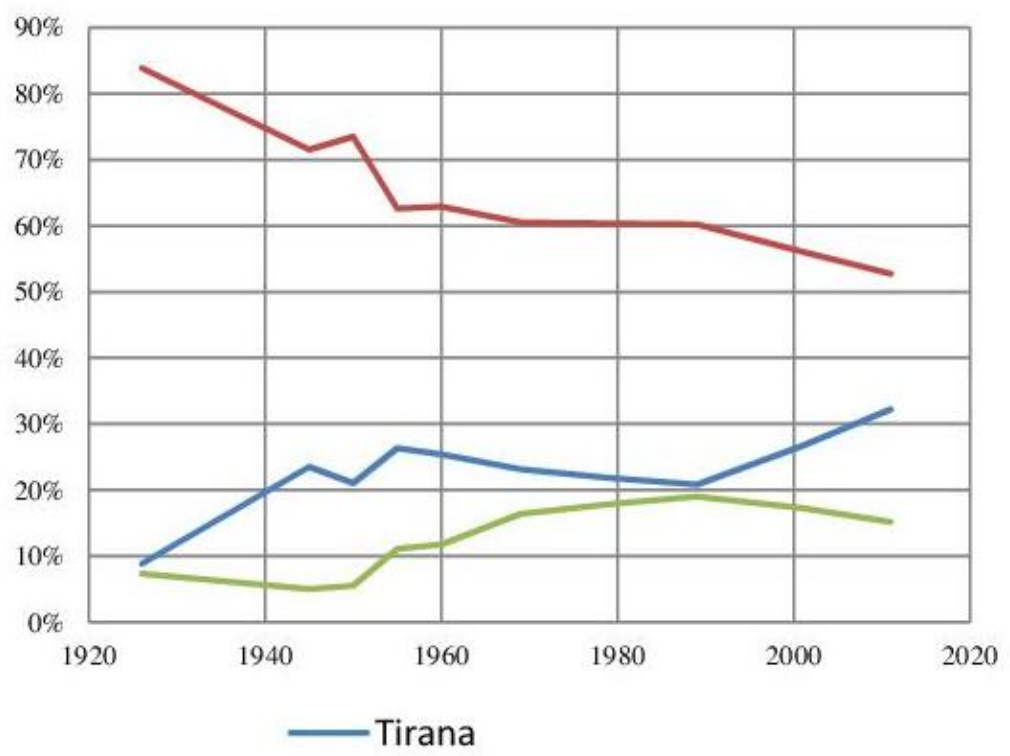

-Villes pré-communistes

-Villes nouvelles

Sources : recensements (compilation de l'auteur) 
7 Nous proposons ici une mesure rétrospective basée sur la liste des localités ${ }^{3}$ considérées urbaines aujourd'hui, en distinguant trois grandes catégories: Tirana, les villes anciennes (26), et les villes nouvelles (44). Nous utilisons également la typologie de territoire de l'Institut national de statistique (Instat) au niveau des communes et municipalités $(2001,2011)$ qui donne une définition du suburbain (Shameti et al., 2014).

8 La montée en puissance de Tirana avant 1960, puis la stagnation (croissance inférieure à celle du pays) de la "métropole discrète » de la période communiste, enfin la reprise de la tendance après 1990 sont spectaculaires. Les villes nouvelles sont un phénomène de la période d'industrialisation, dans les années 1950 essentiellement. Par la suite, elles gagnent des parts dans l'urbain aux dépens de Tirana, mais leur croissance est à peine supérieure à celle de la population albanaise en général. Les villes d'avant le communisme gagnent le plus dans l'absolu ; néanmoins hors la période de stagnation (1960-1990), elles n'ont cessé de perdre au profit de Tirana. Aujourd'hui, la population cumulée de ces 26 villes surpasse à peine celle de Tirana et ses banlieues. Quant aux villages récemment intégrés dans la définition statistique du suburbain de Tirana, ils connaissent une croissance rapide après 1991. Cette imposante immigration vers l'agglomération Tirana-Durrës peut être interprétée à la fois comme un phénomène postsocialiste de rattrapage d'une sous-urbanisation imposée par la stricte application de la doxa de l'équilibre ville-campagne, par la timide mais brutale réintégration dans un monde globalisé, et également par la poursuite d'une tendance amorcée avant 1960, puis gelée jusqu'à 1990, de constitution d'une véritable centralité pour l'Albanie.

Figures 4 à 7 . Croissance de la population urbaine pour quatre périodes de l'histoire contemporaine albanaise.

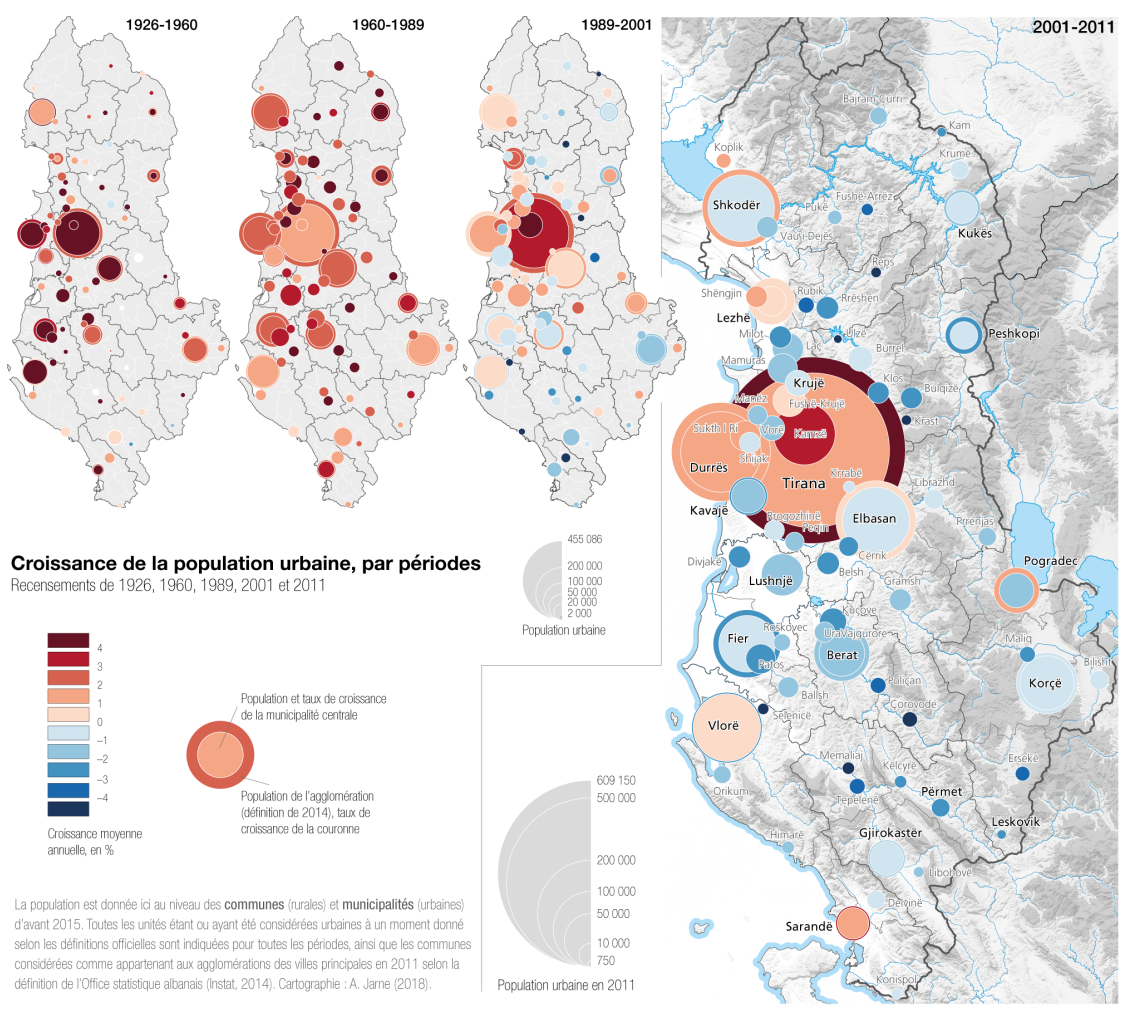




\section{Une mesure de la sous-urbanité}

9 Si les États socialistes, au premier rang desquels l'Albanie, se sont caractérisés par une sous-urbanisation, leurs villes se distinguent également par un gradient d'urbanité inférieur à celui des villes de l'Ouest. Ce dernier est lié à une faible mixité sociale et fonctionnelle ainsi que, tout au moins pour l'Albanie, à la petite taille des unités urbaines, aux fortes restrictions de mobilité - internes ${ }^{4}$ et avec l'étranger ${ }^{5}$ - et au mauvais état des communications, aux différents dispositifs d'assignation spatiale, à la mainmise de l'État sur la production et l'allocation des logements, à une structure centralisée à bien des égards (administration, enseignement supérieur, etc.) ainsi qu'aux différents freins aux interactions sociales déployés par le pouvoir autoritaire. La question de la mobilité, notamment résidentielle, nous intéresse en particulier ici : en effet, elle contribue activement à la structuration des gradients de densité et de diversité déterminant ensemble le niveau d'urbanité. La diversité régionale d'origine des habitants est utilisée ici comme une mesure de la mixité sociale des villes.

On peut dans cette perspective considérer trois types de villes : la capitale, vers laquelle s'orientent les seuls flux significatifs (en particulier avant 1960), les villes existantes qui, lors de leur "socialisation", conservent leur caractère au mieux de centre régional, et les villes nouvelles ou celles qui développent une importante activité industrielle ou minière, dans lesquelles un embryon de mixité se développe qui ne survivra pas à l'effondrement des activités extractives ou industrielles qui ruinera leur maigre attractivité dès 1991. Le gradient d'urbanité propre à Tirana, si son existence est combattue d'un point de vue idéologique car contraire à la doxa de l'égalité des conditions de vie entre la campagne et la ville, demeure cependant une réalité à travers toute la période communiste ${ }^{6}$. La mesure des mouvements de la population durant la période communiste ${ }^{7}$ sur la base du recensement de 2001 montre la faible part des personnes natives d'autres provinces dans les villes albanaises. Si Tirana a en 1989 près de $30 \%$ d'habitants natifs d'ailleurs, les autres villes en comptent en moyenne entre $5 \%$ et $15 \%$, leur taille jouant un rôle, mais davantage encore leur localisation dans des bassins d'immigration interne. Par exemple, la diversité est quasi-nulle dans les bourgades industrielles et extractives des zones montagneuses, qui ne comptent qu'entre $1 \%$ et $5 \%$ de personnes en provenance d'autres régions albanaises. Ces petites villes se sont vidées brutalement à partir de 1997, surtout celles du Nord - comme Bajram Curri, Bulqize, Pukë, Fushe Arrëz, Klos ou Reps - qui ont perdu la moitié ou davantage de leur population dans les quelques années qui ont suivi. À l'opposé, les villes de la plaine côtière ont connu du temps du communisme une immigration supérieure, particulièrement celles situées à proximité de Tirana (Lezhë, Durrës, Laç surtout) ainsi que les coopératives des environs (Bërxull, Kamëz, Sukth, Paskuqan). En termes de mixité (indice de Gini appliqué aux origines régionales), la région capitale (triangle Tirana-Durrës-Laç) se distingue absolument des autres régions d'immigration, ce qui préfigure le développement métropolitain qui marquera l'Albanie post-socialiste.

11 Si on considère le lien entre la mobilité et l'urbanité, on peut encore évoquer le (faible) cosmopolitisme de Tirana qui, durant la période communiste, se limite à la présence temporaire de ressortissants yougoslaves (jusqu'en 1948), puis russes (jusqu'en 1960) et enfin chinois (jusqu'en 1978), «spécialistes » envoyés par les pays amis et partis sitôt ces amitiés dissoutes. Aujourd'hui, la coopération internationale draine ses experts pour des séjours de durées variables, quelques Kosovars ou retraités italiens acquièrent 
des biens immobiliers dans la région capitale, tout cela ne constituant pas une immigration significative. Quant aux migrations de retour, en particulier depuis la crise grecque, qui pourraient signifier un gain de capital économique et social pour les villes, elles s'orientent, semble-t-il, en priorité vers les zones rurales ou périurbaines.

\section{Ruralisation des villes et suburbanisation}

Dès les années 1960, l'aménagement du territoire albanais est marqué par une volonté décentralisatrice privilégiant l'accès à la modernité dans les zones rurales au travers de l'électrification et des télécommunications, de la dotation en infrastructures scolaires, hospitalières, culturelles, de la modernisation de l'agriculture, de la lutte contre les institutions traditionnelles, etc. On pourrait parler d'une volonté d'urbanisation du monde rural. A contrario, on a assisté après 1991 à une «ruralisation " de l'urbain à bien des égards (Çaro, 2013). En effet, dans un contexte de forte croissance quantitative des résidents des agglomérations urbaines dans les années 1990, on assiste au remplacement des nombreux citadins partis vers de plus grandes villes ou à l'étranger par de nouveaux arrivés en provenance de régions rurales et souvent montagneuses. La dilution de certains aspects de l'urbanité est perceptible dans l'apparition d'activités agricoles de subsistance jusqu'au cœur des villes, dans une résurgence de comportements sociaux «traditionnels» dans les banlieues informelles ("retraditionalisation», Lerch, 2013), dans une baisse de qualification de la maind'œuvre et une baisse du niveau moyen de l'éducation, etc. ${ }^{8}$. Pour illustrer l'importance et la manière différenciée dont ce phénomène a touché les villes albanaises, on propose un indice de "ruralisation" de celles-ci pour les deux périodes intercensitaires 1989-2001 et 2001-2011. L'indice se base sur les flux internes et sur une estimation grossière de l'émigration ${ }^{9}$. Il mesure pour chaque municipalité ${ }^{10}$ le bilan des populations urbaines (émigration interne et internationale moins l'immigration urbaine) et celui des populations rurales (immigration rurale), et rapporte la somme des deux à la population de départ. Il se base sur la définition de l'urbain de la typologie territoriale de proposée par l'Instat, qui inclut dans l'urbain d'importants espaces suburbains. Le tableau 1 restitue les valeurs pour les 8 types urbains.

Tableau 1. Migration et ruralisation selon la typologie statistique de l'urbain. Sources : recensements de 1989, 2001 et 2011.

\begin{tabular}{|c|c|c|c|c|c|c|c|c|c|c|c|c|c|c|c|c|c|}
\hline \multirow{3}{*}{$\begin{array}{l}\text { Migration et ruralisation } \\
\text { selon les types urbains }\end{array}$} & \multicolumn{3}{|c|}{ Population } & \multirow{2}{*}{\multicolumn{2}{|c|}{\begin{tabular}{|l} 
Immigration \\
Total (mill.) \\
\end{tabular}}} & & & \multirow{2}{*}{\multicolumn{2}{|c|}{\begin{tabular}{|l} 
Emigration* \\
Total (mill.)
\end{tabular}}} & & & \multicolumn{3}{|c|}{ Solde migratoire* } & & \multirow{2}{*}{\multicolumn{2}{|c|}{\begin{tabular}{|l} 
Ruralisation** \\
Indice
\end{tabular}}} \\
\hline & \multicolumn{3}{|c|}{ Total (milliers) } & & & \multicolumn{2}{|c|}{ Rurale (\%) } & & & \multicolumn{2}{|c|}{ Internat. (\%) } & \multicolumn{2}{|c|}{ Abs. (mill.) } & \multicolumn{2}{|c|}{ Relatif (\%) } & & \\
\hline & 1989 & 2001 & 2011 & $89-01$ & 01-11 & 89-01 & 01-11 & $89-01$ & 01-11 & $89-01$ & $01-11$ & 89-01 & $01-11$ & $89-01$ & 01-11 & $89-01$ & $01-11$ \\
\hline Capitale & 238 & 341 & 418 & 89 & 58 & $43 \%$ & $13 \%$ & 37 & 50 & $91 \%$ & $19 \%$ & 52 & 7 & $22 \%$ & $2 \%$ & 0.10 & 0.02 \\
\hline Centres d'importance nat. & 478 & 501 & 492 & 78 & 41 & $65 \%$ & $40 \%$ & 111 & 101 & $76 \%$ & $56 \%$ & -33 & -60 & $-7 \%$ & $-12 \%$ & 0.28 & 0.18 \\
\hline Centres régionaux & 224 & 227 & 200 & 36 & 17 & $67 \%$ & $46 \%$ & 64 & 70 & $55 \%$ & $27 \%$ & -28 & -53 & $-12 \%$ & $-23 \%$ & 0.34 & 0.30 \\
\hline Centre & 143 & 2 & 118 & 27 & 10 & $91 \%$ & $82 \%$ & 53 & 50 & $41 \%$ & $19 \%$ & -26 & -41 & $-18 \%$ & $-29 \%$ & 0.52 & 0.40 \\
\hline $\begin{array}{l}\text { Suburbain métropolitain } \\
\text { inf. } \\
\text { Suburbain métropolitain }\end{array}$ & 160 & 234 & 286 & 66 & 72 & $72 \%$ & $30 \%$ & 44 & 62 & $60 \%$ & $69 \%$ & 21 & 10 & $13 \%$ & $4 \%$ & 0.46 & 0.14 \\
\hline & 39 & 41 & 94 & 9 & 42 & $73 \%$ & $11 \%$ & 13 & 9 & $51 \%$ & $69 \%$ & -4 & 33 & $-11 \%$ & $79 \%$ & 0.44 & -0.57 \\
\hline Suburbain inf. & 202 & 213 & 194 & 28 & 32 & $80 \%$ & $64 \%$ & 54 & 65 & $57 \%$ & $73 \%$ & -26 & -32 & $-13 \%$ & $-15 \%$ & 0.35 & 0.35 \\
\hline Suburba & 4 & 45 & 4 & 3 & 8 & $81 \%$ & $48 \%$ & 13 & 10 & $74 \%$ & $87 \%$ & -8 & -3 & $-18 \%$ & $-6 \%$ & 0.34 & 0.24 \\
\hline Tira & 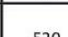 & 715 & בו & 100 & 10 & -50 & 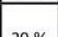 & 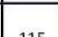 & & 70 & ron & & & 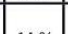 & 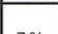 & 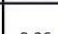 & 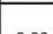 \\
\hline
\end{tabular}

* Estimations. ** Calcul de l'indice : ((immigration rurale - immigration urbaine) + émigration) / population de départ 

une forte immigration rurale, avec l'idée que celle-ci subit une perte de «capital urbain », et qu'elle devra « investir » pour re-fabriquer de l'urbanité avec sa nouvelle population d'origine rurale (régler les problèmes de formation, de paupérisation, d'intégration, etc.). La ville de Burrel ( $35 \mathrm{~km}$ au nord-est de Tirana) en est l'exemple : 5500 environ de ses 11500 habitants ont quitté la ville entre 1989 et 2001 (2/3 vers l'étranger); dans le même temps, elle a accueilli 5000 personnes en provenance de communes rurales pour 150 de municipalités urbaines, ce qui donne un indice exceptionnellement élevé de 0,90 ((5500-150+5000)/11500). À l'opposé, Tirana (à l'intérieur de sa " ligne jaune $\left.{ }^{11}\right)$, grâce à une émigration modérée et une immigration largement urbaine (à $57 \%$ ), connait la plus faible "ruralisation» d'Albanie: elle accueille 38000 ruraux, et gagne 14000 urbains (37 000 départs, 51000 arrivées), ce qui donne un indice de 0,1 . Le profil migratoire, et donc la "ruralisation», parait déterminé par la position dans la hiérarchie urbaine et la position géographique, sur un gradient Tirana - plaines côtières - régions périphériques.

Figure 8. Illustration de la « ruralisation » des villes albanaises, les troupeaux paissent et cet homme tond un mouton à deux pas de la gare centrale (aujourd'hui détruite) de Tirana).

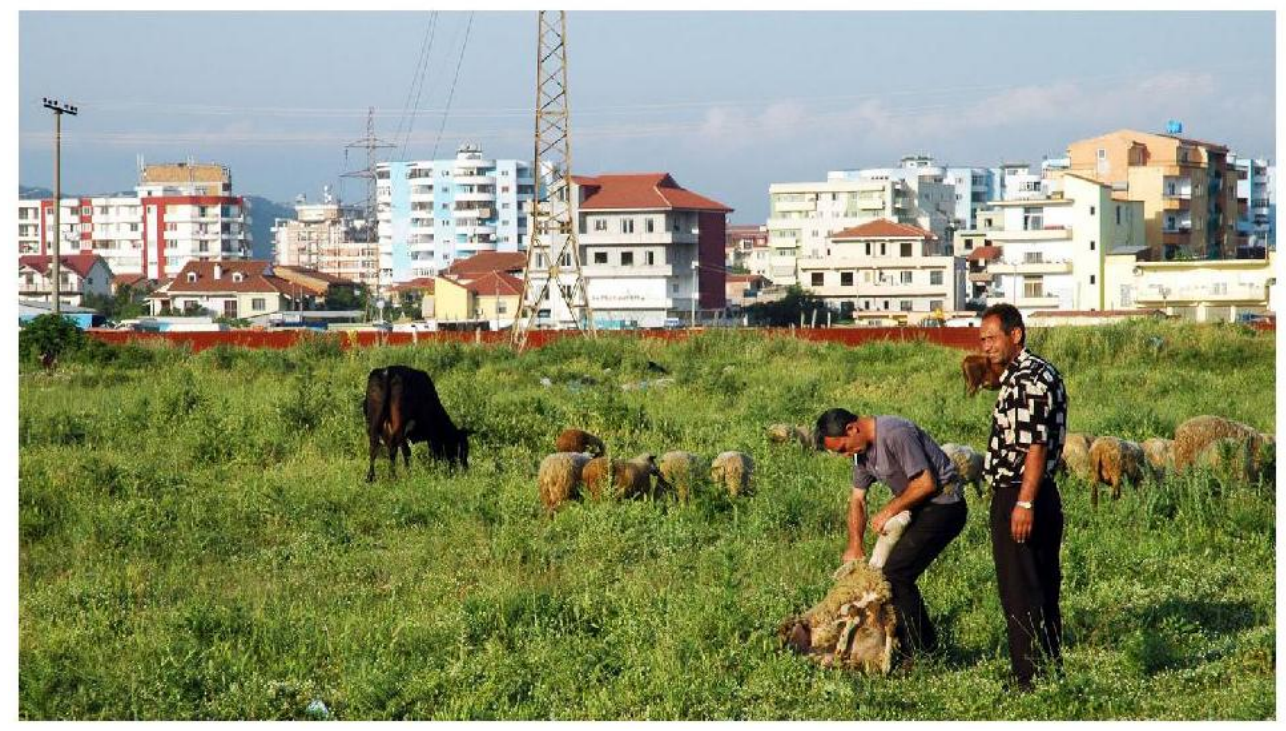

Photo : A. Jarne, 2008

L'immigration entre 1989 et 2001 représentait $37 \%$ de la population de 1989 à Tirana et à Lezhë, $30 \%$ à Durrës, et en général entre $10 \%$ et $20 \%$ dans les autres villes, à l'exception de certains centres de régions enclavées qui ont accueilli une importante population rurale eu égard à leur taille (Peshkopi $26 \%$, Ersekë $28 \%$, Gramsh $34 \%$, Ballsh $36 \%$ et Burrel $44 \%$ ). Dans les grandes villes de la plaine côtière, une part importante de l'immigration s'établit au-delà de la ligne jaune, à des niveaux élevés dans les nouvelles banlieues de Tirana (Kashar $43 \%$, Bërxhull $60 \%$, Kamëz $225 \%$ et Paskuqan $322 \%$ ), de Durrës (Rashbull 53 \%, Sukth $62 \%$ ) de Lezhë (Kolsh 55 \%, Shënkoll $36 \%$ ) et de Shkodër (Rrethinat $31 \%$ ). Cette immigration a une structure en cascade, presque exclusivement rurale (91\%) dans les centres locaux, mixte dans les villes moyennes ( $65 \%$ rurale) et à dominante urbaine à Tirana ( $43 \%$ rurale). Quant à l'émigration, elle est de $15 \%$ à Tirana et culmine à $37 \%$ dans les centres locaux, avec 
des taux records dans les anciennes villes nouvelles implantées dans les régions montagneuses et désertées par la moitié ou plus de leurs habitants (Burrel, Pukë, Bajram Curri, etc.). La part de cette émigration à destination de l'étranger décroît avec la taille des villes : de $90 \%$ à Tirana à $40 \%$ dans les centres locaux. La résultante en est que la ruralisation touche très fortement les petites villes enclavées (forte émigration, immigration essentiellement rurale) et les nouvelles banlieues informelles (très forte immigration rurale); les grands centres, quant à eux, sont le moins touchés (émigration moindre, immigration mixte). Si ces derniers gagnent même de la population dans la première décennie qui suit la chute du régime, ce n'est désormais plus le cas: leur solde migratoire rendu fortement négatif par le tarissement de l'immigration (elle diminue de moitié entre les deux derniers recensements), conjugué à la poursuite de l'émigration, n'est plus compensé par un solde naturel désormais en légère baisse. Les enjeux de revitalisation des centres moyens, voire de simple survie pour les petites villes dépourvues de leur fonction industrielle, sont donc réels. Mais un autre enjeu concerne les grands centres, et Tirana au premier chef : si cette ruralisation est moindre dans les périmètres municipaux, l'immigration rurale est réelle et se concentre à l'extérieur des lignes jaunes, dans les banlieues informelles apparues dans les années 1990, produisant ainsi une ségrégation plus immédiatement observable qu'ailleurs, parce que traduite dans la statistique officielle.

Dans ces grandes «villes duales» (Pojani 201; Prato 2016) du début des années 2000 que sont devenues Tirana et, dans une moindre mesure, Shkodër et Durrës, débute alors la seconde phase d'urbanisation post-socialiste, à savoir un mouvement nouveau du centre vers la périphérie des villes. Mais la très forte tendance à la métropolisation ${ }^{12}$ fait que ce phénomène est surtout spectaculaire à Tirana. Sur 45000 personnes qui viennent s'établir dans les nouveaux quartiers de la périphérie de Tirana (à Dajt, Vaqarr, Kashar ou Farkë) entre 2001 et 2011, 53 \% viennent de la ville-centre (dans la décennie précédente, pour les principales extensions de Tirana - communes de Kamëz et Paskuqan - seuls $2 \%$ des quelque 23000 arrivants venaient du centre). L'indice de ruralisation fortement négatif des communes «suburbaines supérieures" indique ce mouvement. En lieu et place des maisons auto-construites d'un, voire deux logements des années 1990, sont érigés depuis le milieu des années 2000 de grands immeubles serrés, dotés d'accès, d'équipements et d'espaces publics souvent de médiocre qualité.

\section{Tirana, un cas à part}

L'Albanie est un pays marqué par de profonds régionalismes. A l'échelle nationale, les différences culturelles entre le nord et le sud, loin d'avoir été gommées par l'histoire récente, demeurent marquantes à plus d'un titre (De Rapper, 2004). Capitale récente (1920), Tirana, ville secondaire alors, a été choisie pour sa position centrale, d'un point de vue géographique et symbolique : elle est appelée alors à devenir la métropole de l'Albanie nouvelle, surplombant les tensions interrégionales, les loyautés claniques, cœur de l'État et foyer de la modernisation de la Nation. Dans cet ordre d'idée, l'équilibre et la mixité des représentations régionales dans la capitale sont des enjeux centraux de l'urbanité spécifique qui s'est développée au cours du siècle écoulé depuis l'Indépendance. Envisagée ainsi, l'histoire de la structure de l'apport migratoire exclusivement national qui fait passer la bourgade rurale de 12000 habitants à l'aire métropolitaine avoisinant le million d'habitants souligne cette idée: Tirana est un 
« miroir » de l'Albanie et de ses fractures, en particulier. La succession des mouvements de population et la manière dont ceux-ci structurent l'espace social dans l'aire métropolitaine Tirana-Durrës est illustrée par un tableau qui montre le basculement de l'origine des migrations vers la capitale et une série de trois cartes qui montre la ségrégation qui en résulte à destination. Les origines ont été considérées pour cinq grandes régions pertinentes du point de vue de l'histoire des migrations internes albanaises : i) la région capitale, à savoir les préfectures de Tirana et Durrës; ii) la région Nord, préfectures de Shkodër et Lezhë ; iii) la région Nord-Est (Dibër et Kukës); iv) la région centrale (Elbasan, Fier et les districts de Berat et Kucovë) ; v) le Sud (Vlorë, Korçë, Gjirokastër et le district de Skrapar). Une forte migration des régions montagneuses $\mathrm{du}$ sud et du nord-est, selon des modalités et des temporalités différentes, s'est faite en direction de Tirana, alors que les périmètres nord et centre ont plus largement connu des migrations internes.

Tableau 2. Parts de l'immigration vers l'aire métropolitaine Tirana-Durrës en provenance des différentes régions d'Albanie.

\begin{tabular}{|l|c|c|c|}
\hline $\begin{array}{l}\text { Vers aire métropolitaine Tirana-Durrës } \\
\text { en provenance de... }\end{array}$ & $\begin{array}{c}\text { Période communiste } \\
\text { (lieu de naissance- } \\
\text { domicile en 1989) }\end{array}$ & $\begin{array}{c}\text { Années 1990 } \\
\text { (domicile en 1989- } \\
\text { domicile en 2001) }\end{array}$ & $\begin{array}{c}\text { Années 2000 } \\
\text { (domicile en 2001 } \\
\text {-domicile en 2011) }\end{array}$ \\
\hline Région Tirana-Durrës, hors agglomération & $16 \%$ & $8 \%$ & $8 \%$ \\
\hline Région Nord (Shkodër, Lezhë) & $7 \%$ & $11 \%$ & $12 \%$ \\
\hline Nord-Est (Dibër, Kukës) & $13 \%$ & $35 \%$ & $32 \%$ \\
\hline Centre (Elbasan, Fier, Berat, Kuçovë) & $18 \%$ & $21 \%$ & $28 \%$ \\
\hline Sud (Vlorë, Korçë, Gjirokastër, Skrapar) & $47 \%$ & $24 \%$ & $20 \%$ \\
\hline
\end{tabular}

Sources : recensements de 2001 et 2011

17 La période communiste, surtout à son début, présente cette caractéristique de la forte prééminence du Sud dans les migrations vers la capitale, au travers à la fois d'une forte immigration urbaine et d'une importante immigration rurale. Ailleurs, au centre en particulier, l'immigration est essentiellement urbaine. Ces constats s'expliquent notamment par i) la forte représentation des gens du sud, orthodoxes en particulier, dans les élites communistes; ii) le bouclage du régime traditionnel de migration vers l'étranger propre au Sud et sa réorientation vers la capitale, aussi en raison iii) de l'absence de centralité urbaine de premier ordre; iv) la difficulté pour le Sud et son agriculture extensive de répondre aux besoins d'une population à fort croît démographique. Cette force de l'immigration du Sud dans la capitale durant le communisme pose la question d'une forme de déséquilibre culturel propre à cette période. Ces inégalités entre Nord et Sud (parfois ramenées à l'opposition entre Gègues et Tosques ${ }^{13}$ ) durant le communisme sont avérées, qu'il s'agisse de l'histoire du Parti et de ses élites, de la langue officielle telle que fixée à cette époque, et largement dominée par les apports tosques, etc. Au demeurant, on observe au niveau économique plutôt une réduction des inégalités économiques entre les régions au fil des ans ${ }^{14}$. Pour ce qui concerne les déséquilibres dans l'apport migratoire de Tirana, il semblerait que la prééminence du Sud ait été surtout un phénomène de l'après-guerre et se soit érodé avec le temps. La segmentation en cohortes montre en effet que pour la municipalité (ancienne) de Tirana, la part des immigrés (naissance-1989) du Sud est de $59 \%$ chez les 66-75 ans en 2001 (plus susceptibles d'avoir émigré dans les années 1950), contre seulement $36 \%$ chez les $36-45$ ans (plus susceptibles d'avoir migré dans les années 
1980). Toujours est-il que la Tirana communiste demeure marquée par cet apport migratoire bien spécifique.

La décennie qui suit la fin du socialisme voit un basculement de ces structures : si le Sud se tourne à nouveau très largement vers l'émigration (souvent sous la forme de "champs migratoires" avec la Grèce voisine ${ }^{15}$ ) de même que la bande côtière (préférentiellement vers l'Italie), les régions jusque-là peu représentées dans la capitale du Nord-Est (Dibër, Kukës, Has et Tropojë) se déversent massivement sur l'agglomération. La grande majorité de ces nouveaux arrivants sont des ruraux qui s'installent dans les nouvelles banlieues « informelles » au nord de Tirana, à Paskuqan et Kamëz essentiellement. L'autre provenance de départs massifs vers Tirana est constituée des montagnes de Dangellia, Ostrovica, Skrapari et Opari au sud-est de Berat. Cette seconde période prend la forme d'un véritable exode des montagnes, comme en réaction à trois décennies de rétention rurale. Au contraire, la période plus récente voit reprendre les déplacements entre villes, en direction de Tirana d'abord, mais aussi entre et vers d'autres villes importantes. Les villes de Peshkopi et de Kukës perdent chacune environ 10000 habitants au profit de Tirana, et les ex-villes nouvelles industrielles des régions montagneuses septentrionales (Pukë, Bulqizë, Burrel), mais aussi plus au sud (Librazhd, Gramsh, Tepelenë et Permët), se vident littéralement vers Tirana.

Figure 9. Densité de peuplement et périodes de construction dans Tirana et sa proche banlieue.

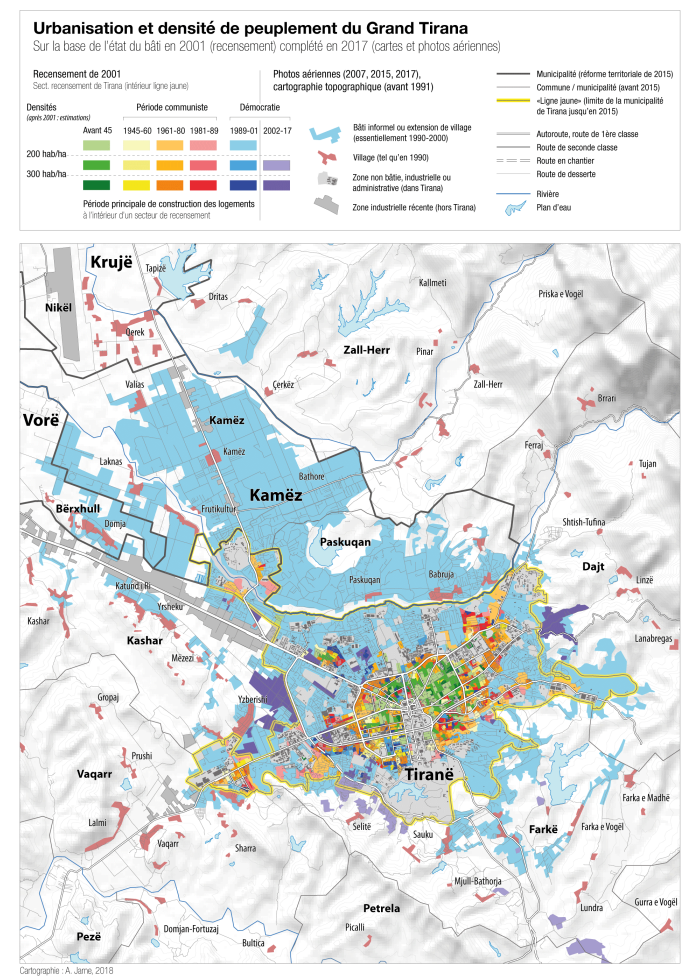




\section{La ségrégation socio-spatiale dans l'aire métropolitaine}

19 La ségrégation socio-spatiale dans l'aire métropolitaine Tirana-Durrës constitue une forme d'enregistrement des séquences successives de son urbanisation, avec une forte différentiation spatiale dans la distribution, outre des origines régionales, des niveaux d'éducation, des types de logements ou de l'accès à l'emploi ${ }^{16}$. Les cartes qui suivent illustrent la manière dont cette immigration se répartissait vers $2000^{17}$ dans l'agglomération en fonction de son origine.

La première carte dessine en négatif le bassin d'immigration que constitue la plaine de Tirana, limité sur trois de ses flancs par les reliefs et trouvant un débouché naturel pour son extension au nord-ouest vers Fushë Krujë. La seconde carte montre à quel point l'immigration du Sud est concentrée au cœur de la ville où elle représente souvent à elle seule plus de la moitié des immigrés, ainsi que dans les cités satellites anciennes (par exemple, dans le quartier du Combinat Textile Staline vers Yzberish au sud-est, plus de $60 \%$ des immigrés proviennent du Sud). Au nord, la petite ville (déclarée comme telle dans les années 1980) de Kamzë était une grande coopérative agricole largement peuplée d'immigrants du Sud, autour de laquelle s'est étalée dans les années 1990 une mer de maisonnettes peuplée d'immigrants venus du Nord-Est. Cette vague d'immigration des années 1990 vers Tirana, souvent décrite car exceptionnelle dans le contexte européen, voit arriver environ 150000 nouveaux résidants entre 1989 et 2001, dont près de la moitié en provenance du Nord-Est, soit $14 \%$ de la population totale de l'aire métropolitaine en 2001. La concentration est très forte à Kamëz et Paskuqan, où les gens fraîchement arrivés du Nord-Est représentent alors plus de $55 \%$ de la population et $70 \%$ des immigrés, contre seulement $10 \%$ de la population et $30 \%$ des immigrés à Tirana. Cette population du Nord-Est se concentre dans l'habitat dit informel. Les rares quartiers où s'est construit durant cette période de l'habitat plus central et plus dense, dans le périmètre de l'ancien Blok $^{18}$ en particulier, n'hébergent quasiment aucune population du Nord-Est. 
Figures 10 à 12. Immigrants dans l'agglomération de Tirana, selon la région de naissance.
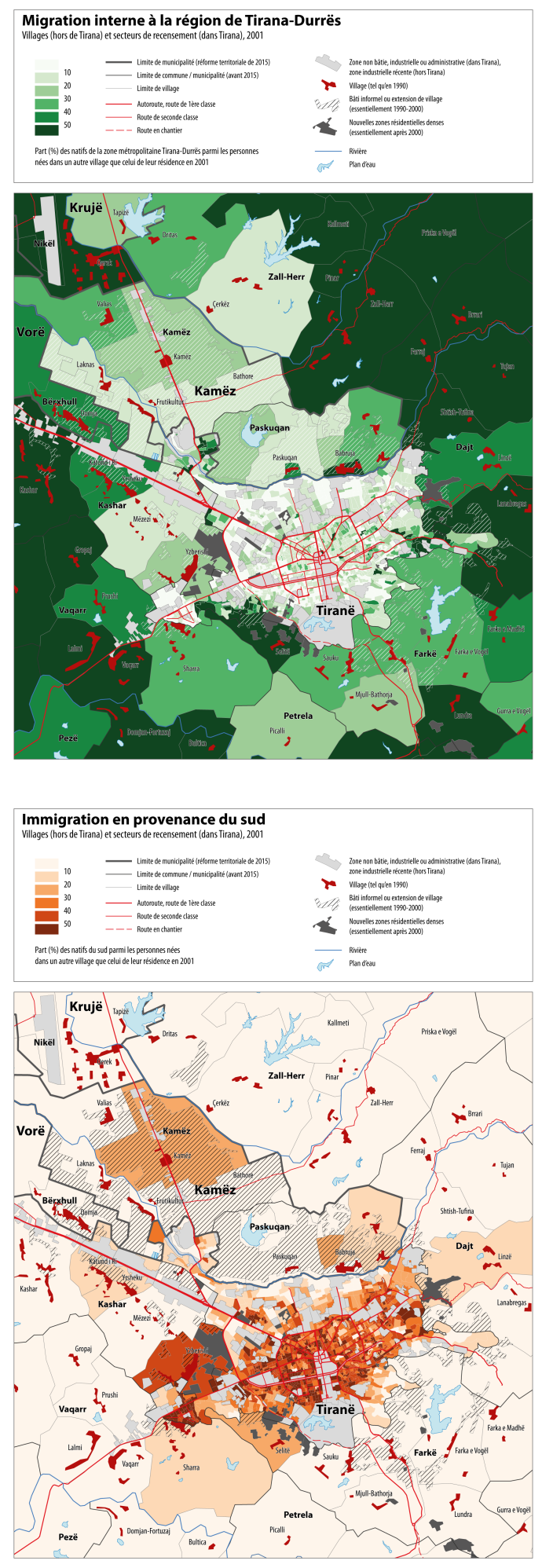


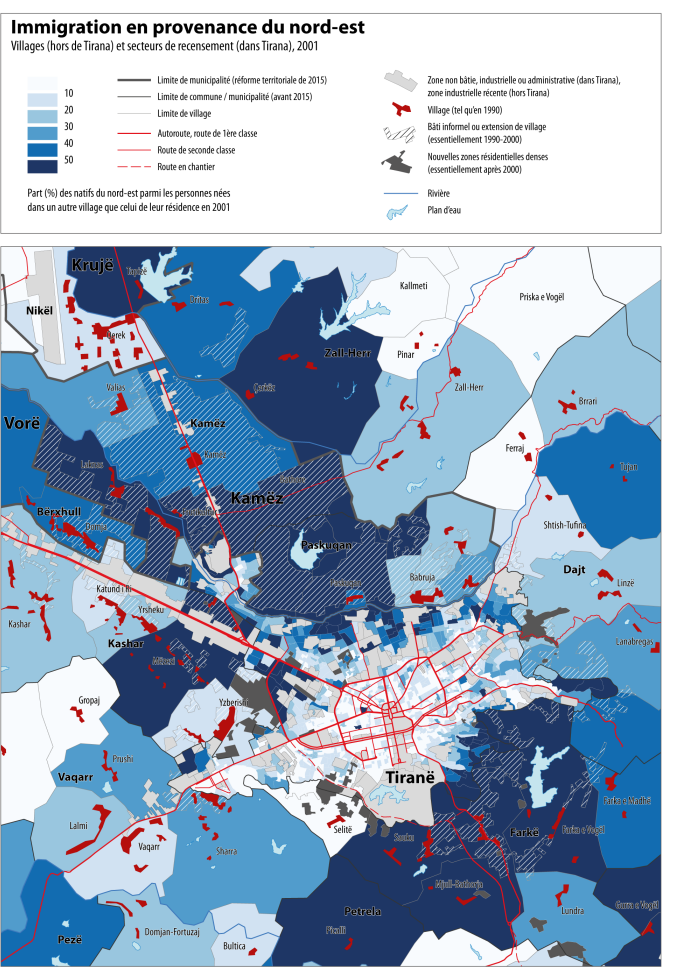

Tableau 3. Immigrants à Tirana et dans ses banlieues, par périodes et régions de provenance, au niveau des communes/municipalités d'avant 2015.

\begin{tabular}{|c|c|c|c|c|c|c|c|c|c|c|c|c|c|c|c|c|c|c|}
\hline & \multicolumn{9}{|c|}{ Période 1989-2001 } & \multicolumn{9}{|c|}{ Période 2001-2011 } \\
\hline & 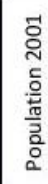 & 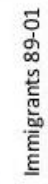 & 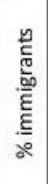 & 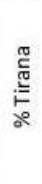 & 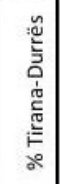 & $\begin{array}{l}\text { 무 } \\
\text { 2 } \\
\text { o }\end{array}$ & 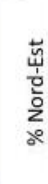 & 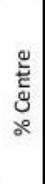 & $\begin{array}{l}\text { Dे } \\
\text { ऽొ }\end{array}$ & 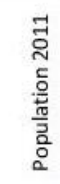 & 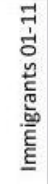 & 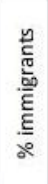 & 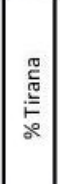 & 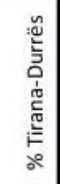 & $\begin{array}{l}\text { o } \\
2 \\
20 \\
\text { de }\end{array}$ & 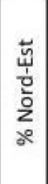 & 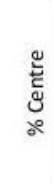 & $\begin{array}{l}\text { בे } \\
\text { ปे }\end{array}$ \\
\hline Tirana & 341 & 89 & 26 & - & 18 & 10 & 28 & 19 & 25 & 418 & 58 & 14 & & 12 & 12 & 17 & 33 & 26 \\
\hline Kashar (est) & 17 & 5 & 27 & 4 & 17 & 5 & 34 & 13 & 27 & 43 & 20 & 46 & 47 & 5 & 4 & 10 & 17 & 16 \\
\hline Farkë (sud) & 7 & 2 & 31 & 3 & 24 & 1 & 61 & 7 & 4 & 23 & 10 & 46 & 52 & 9 & 2 & 10 & 15 & 13 \\
\hline Dajt (ouest) & 8 & 2 & 18 & 8 & 31 & 12 & 32 & 7 & 10 & 20 & 11 & 55 & 63 & 5 & 4 & 10 & 8 & 10 \\
\hline Kamëz (nord) & 44 & 22 & 50 & 2 & 6 & 11 & 74 & 3 & 3 & 67 & 21 & 32 & 37 & 7 & 10 & 39 & 5 & 2 \\
\hline Paskuqan (n.) & 21 & 11 & 53 & 1 & 8 & 5 & 70 & 6 & 10 & 37 & 12 & 32 & 33 & 12 & 6 & 39 & 6 & 4 \\
\hline
\end{tabular}

Source : recensements de 2001 et 2011

21 Au développement informel de la première décennie postcommuniste au nord et, dans une moindre mesure, au sud de Tirana a répondu dès le milieu des années 2000 un développement beaucoup plus compact essentiellement à l'ouest et à l'est, à Dajt, sur les flancs de la montagne du même nom. Le tableau 3 donne les provenances des immigrés pour Tirana et les cinq anciennes communes qui abritent ses principales banlieues. On y voit, d'une part, à quel point les mouvements centrifuges démarrent après 2000 et d'autre part, leur concentration à l'est, à l'ouest et au sud. Le cas le plus emblématique est sans doute la plaine d'Yzberish (dans l'ancienne commune de Kashar), qui n'avait que partiellement été conquise par l'habitat informel. De grands ensembles d'une dizaine d'étages sont venus prendre place d'abord dans les interstices, puis sont venus remplacer les petites maisons construites une vingtaine d'années 
auparavant. La moitié de la nouvelle population vient de Tirana, le reste plutôt du centre et du sud du pays. Des habitants de Tirana vont également s'établir à Kamëz et Paskuqan, mais celles-ci restent dominées par l'immigration de Dibër, Has, Kukës et Tropojë, alors que l'immigration du Sud y est nulle.

Les années du début du communisme ont donc marqué durablement la composition du centre de Tirana, tandis que celles qui ont suivi la chute du communisme ont forgé la composition de ses banlieues, le mécanisme des migrations en chaîne y étant pour beaucoup dans la perpétuation des structures de la représentation régionale héritées de ces deux moments d'intenses migrations.

Figure 13. Vue typique du tissu bâti « informel » qui s'est développé de manière rapide et désordonnée à la fin des années 1990 et au début des années 2000 surtout dans les banlieues nord, ici à Kamzë.

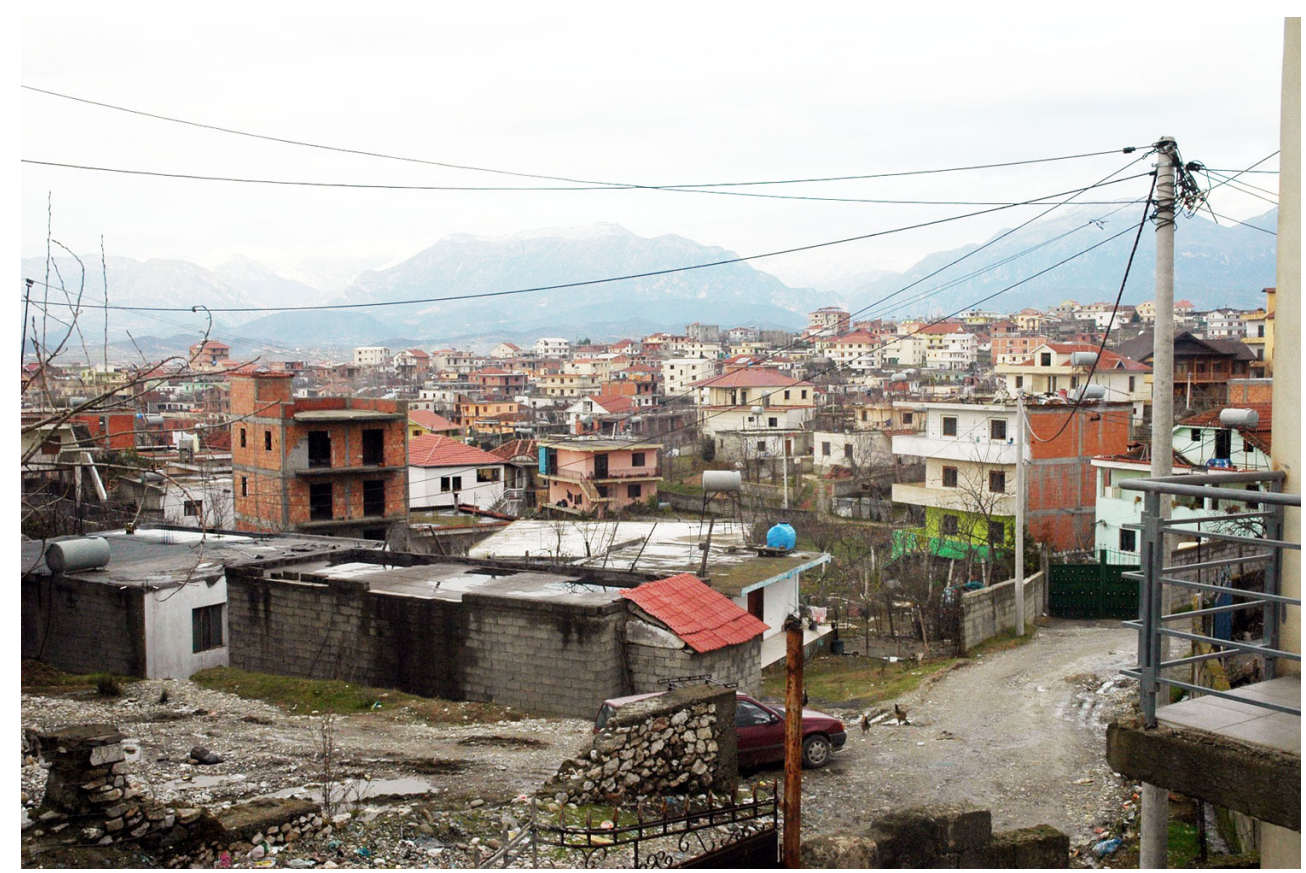

Photo : A. Jarne, 2010 
Figure 14. Vue du centre (ancien Blok) vers le sud-ouest. Au premier plan, les immeubles de 4-5 étages typiques du communisme, au second plan un quartier encore proche du centre et dense des années 90 , et à l'arrière-plan, les développements urbains récents qui colonisent désormais les flancs des collines autour de Tirana, à l'est en particulier.

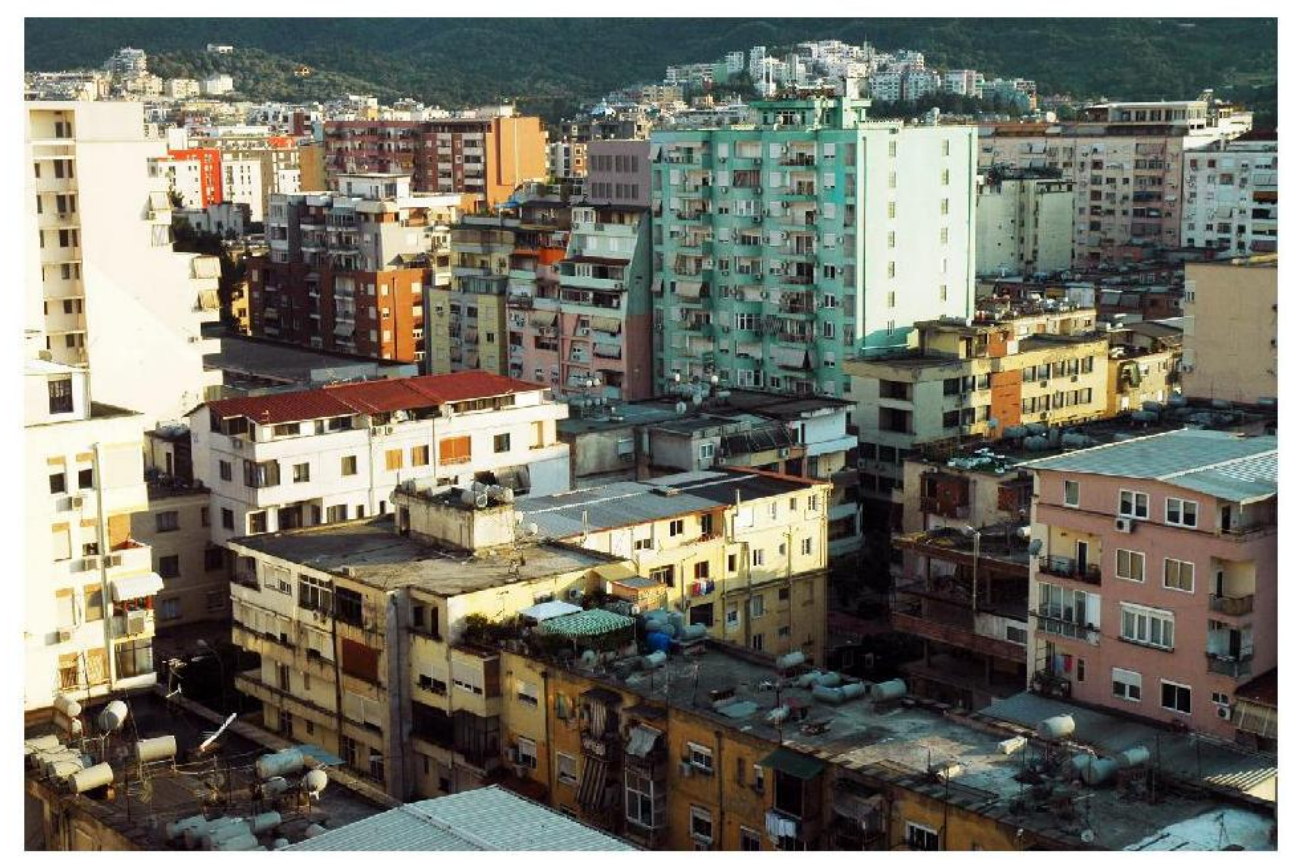

Photo : A. Jarne, 2017

\section{La densité}

Dans l'idée que les gradients d'urbanité découlent largement de l'association de la diversité et de la densité, on donne rapidement la mesure de celle-ci. Aussi et surtout parce qu'il nous paraît que dans le cas de Tirana, la densité articule la relation entre l'exclusion sociale et le projet urbain. Dans ces quartiers d'immigration, bien que proches du centre, la faible densité, l'absence de mixité fonctionnelle et la fragmentation du foncier posent les problèmes du coût relatif de l'équipement, de la non-rentabilité d'une desserte par les transports publics, de la difficulté du déploiement de projet immobilier d'envergure, etc. L'exclusion des nouveaux venus, ruraux du Nord, peut ainsi reposer sur un discours fondé sur une rationalité urbanistique.

On observe très clairement une augmentation progressive des densités au cours du temps, avec des niveaux moyens inférieurs à 180 hab./ha pour les quartiers anciens, puis des valeurs croissant durant la période communiste, de 300 hab./ha après-guerre à souvent plus de 400 dans les années $1980^{19}$. Les quartiers plus mixtes - bâti ancien clairsemé d'immeubles plus récents - ont en général des densités avoisinant les 200 hab./ha. Après 1991, ces valeurs chutent à des niveaux historiquement bas lorsque les populations venues du Nord investissent les zones agricoles, maraîchères (Allias), certains parcs et forêts (Lapraka), ainsi que les interstices de moindre importance laissés libres à l'intérieur d'un périmètre municipal alors généreusement doté en espaces ouverts. Ces constructions dites "informelles» sont des maisons familiales autoconstruites avec cour ou jardin, les densités y sont de l'ordre de 80 hab./ha. La 
taille moyenne des immeubles évolue de 1,6 logements/immeuble avant-guerre à 3,3 avant 1960, 5,9 entre 1960 et 1980, 4,3 dans les années 80 et 1,7 dans les années 1990. Lorsque, à partir des années 2000 , le secteur de la construction redémarre ${ }^{20}$, les principaux quartiers d'habitation sont érigés au-delà de la seconde couronne et des limites municipales d'alors, à plus de trois kilomètres du centre, sur les territoires des anciennes communes de Kashar, Vaqarr, Farkë et Dajt. Les deux ensembles les plus remarquables sont à l'est à Linzë (Dajt) et à l'ouest à Yzberish (Kashar). En termes de densité de peuplement, on observe donc un centre relativement lâche, une première ceinture plus compacte datant du communisme, un semis de petites maisons autoconstruites parsemées des quelques cités satellites héritées (Lapraka, Shkoza, Kombinat et Kino Studio) et, au-delà, les nouveaux ensembles récents à très forte densité. Quelques projets résidentiels significatifs se déploient également à l'intérieur de l'ancienne ligne jaune, dans des friches industrielles (partie sud du combinat de tracteurs Enver Hoxha) ou dans des espaces encore ouverts, comme l'ancien aérodrome de Tirana. Les potentiels de densification sont réels, mais la difficulté de réhabiliter les zones industrielles (coûts, pollution) ou de remplacer le bâti épars (questions foncières) met sous pression les espaces publics, comme en témoignent les constructions récentes sur les anciens terrains de sport des Partisans. Le développement d'une liaison périphérique orientale, héritage partiel de l'ancien projet de $1989^{21}$ connectant les routes de Durrës à celle de Kavaja et tout récemment à celle d'Elbasan, favorise le développement de cités dortoirs sur une gamme allant des hautes densités souséquipées d'Yzberish à des « gated communities » de luxe à la sortie sud de la ville ${ }^{22}$.

Le tableau 4 fait le lien entre la ségrégation selon les origines et la densité du bâti mesurée par le nombre moyen de logements dans l'immeuble de résidence. Les densités s'échelonnent selon le double gradient des contextes urbains plus ou moins centraux et des origines régionales.

Tableau 4. Densité du bâti au lieu de résidence, selon l'origine régionale (nombre moyen de logements de l'immeuble de résidence selon lieu de naissance des habitants (10 ans et plus), en 2001, pour les 6 principales communes de l'agglomération de Tirana).

\begin{tabular}{|l|c|c|c|c|c|c|}
\hline \multirow{2}{*}{$\begin{array}{l}\text { Lieu de } \\
\text { résidence } \\
\text { en 2001 }\end{array}$} & \multicolumn{5}{|c|}{ Lieu de naissance } & nord-est \\
\cline { 2 - 7 } & toutes origines & Tirana-Durrës & nord & centre & \\
\hline Tiranë & 2,35 & 2,37 & 2,24 & 1,78 & 2,50 & 2,48 \\
\hline Kashar & 1,28 & 1,29 & 1,36 & 1,25 & 1,30 & 1,29 \\
\hline Farkë & 1,23 & 1,25 & 1,22 & 1,22 & 1,19 & 1,29 \\
\hline Dajt & 1,34 & 1,33 & 1,29 & 1,27 & 1,52 & 1,50 \\
\hline Kamëz & 1,32 & 1,64 & 1,24 & 1,18 & 1,31 & 1,72 \\
\hline Paskuqan & 1,24 & 1,41 & 1,17 & 1,15 & 1,32 & 1,27 \\
\hline Total & $\mathbf{2 , 1 5}$ & $\mathbf{2 , 2 2}$ & $\mathbf{1 , 9 9}$ & $\mathbf{1 , 4 8}$ & $\mathbf{2 , 3 7}$ & $\mathbf{2 , 3 9}$ \\
\hline
\end{tabular}

Source : recensement de 2001. 
Figures 15 à 17. Etalement urbain dans la plaine d'Yzberish à l'ouest de Tirana.
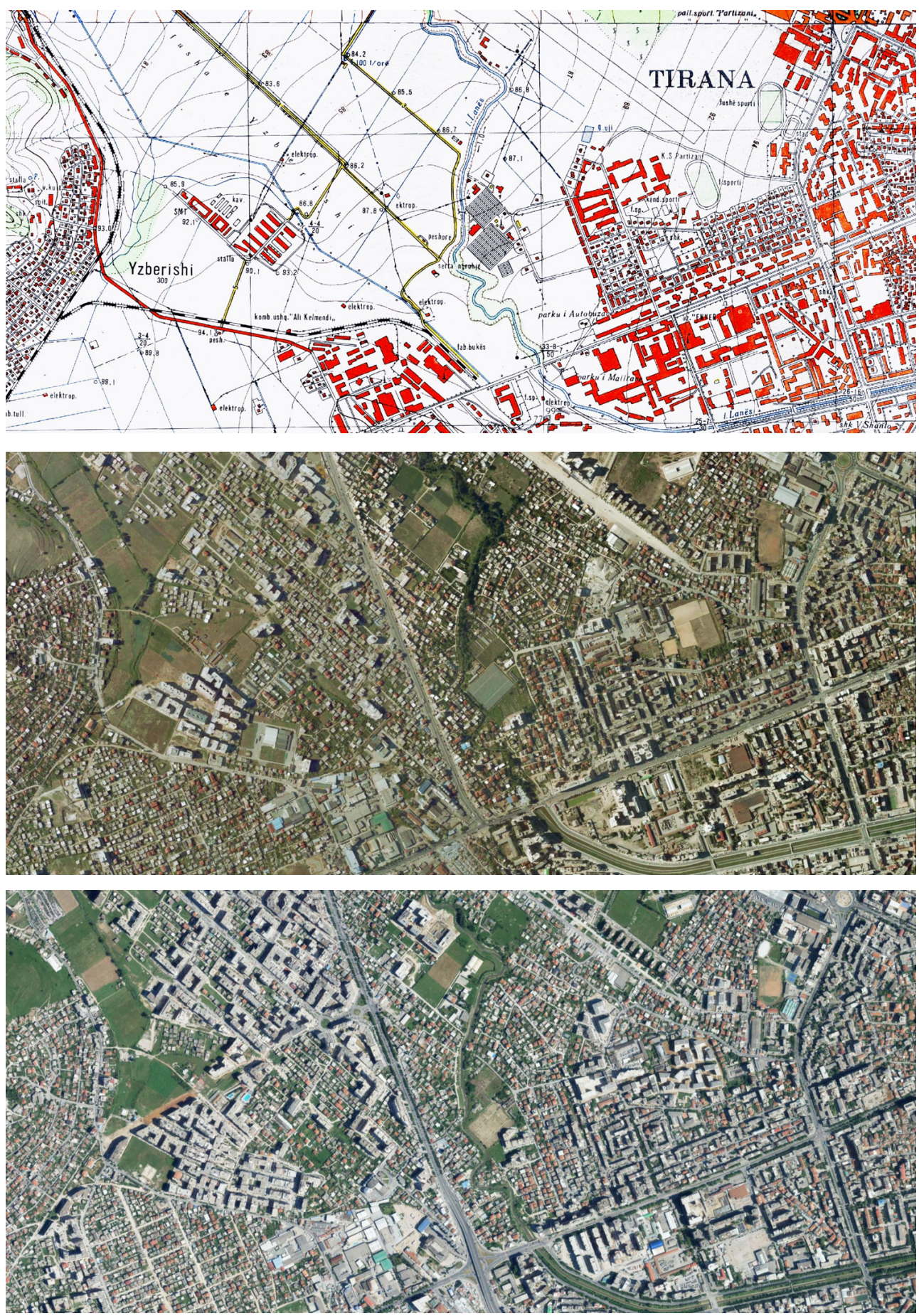

(c) Autoriteti Shtetror për Informacionin Hapsinor (ASIG)

Cartes au $1: 10000$ de 1984 ; photographies aériennes de 2007 et 2018

\section{Conclusions : les dimensions de l'exclusion et le projet urbain}

La brutale immigration survenue après 1991 a bouleversé la vision que Tirana se faisait de son développement. Les plans directeurs de la fin du communisme (1989) 
prévoyaient un périphérique extérieur, conçu aussi comme limite du développement des 15 années à suivre. Il prévoyait aussi le prolongement de l'axe principal, le boulevard des Martyrs de la Nation, vers le nord, à travers Paskuqan et Kamëz vers l'aéroport, Durrës et Shkodër, en faisant l'accès principal à la ville. Par la suite, les planifications directrices ont eu à s'adapter à la nouvelle donne : les immenses secteurs informels. Celle menée au début des années 2000 (2002) au niveau du "Grand Tirana » reprenait l'idée du périphérique externe, y intégrant le sud de Paskuqan. L'idée du prolongement du boulevard central était également reprise, mais pour le poursuivre cette fois-ci en une sorte de périphérique supplémentaire ceignant les immenses zones informelles de Paskuqan et Kamëz pour venir se brancher sur la route de Durrës à Bërxull, intégrant les banlieues défavorisées dans l'agglomération par-delà la barrière naturelle que constitue la rivière de Tirana. Le tronçon de la route de Durrës entre le centre et Bërxull était, quant à lui, voué à accueillir prioritairement des transports publics. Quinze ans plus tard, c'est-à-dire l'horizon que s'était fixé cette stratégie, rien de tout cela n'a vu le jour. Bien au contraire, les banlieues nord paraissent plus enclavées que jamais, suscitant cette terminologie de ville "duale». La réforme territoriale de 2015 a institutionnalisé l'exclusion de Kamëz, en faisant une municipalité distincte de celle de Tirana $^{23}$. Cela se répercute dans les projets de territoire récents pour Tirana dont la cartographie et l'imagerie 3D peuvent désormais «omettre » ces quartiers, dans un courant général qui voit les problématiques sociales marginalisées au profit de considérations générales sur la durabilité, l'environnement, etc. Désormais dotée d'autoroutes vers l'ouest et vers le sud (Elbasan), Tirana tourne résolument le dos à sa banlieue nord. D'une certaine manière, pour la Municipalité de Tirana, des questions urbanistiques parmi les plus délicates se sont trouvées ainsi évacuées.

Commentée dans le sens d'une ségrégation économique, la césure Tirana-Kamëz a aussi clairement une signification identitaire et politique, qui a trait à cette "question fondamentale de la réalité albanaise, celle de la prise en compte de la diversité régionale dans la formation de la nation et du territoire national» (De Rapper 2004, p.640). Cette fracture se retrouve également dans les comportements électoraux, poussant des observateurs à assimiler la réforme territoriale à du "gerrymandering ${ }^{24}$ ", dans le sens où, par exemple, l'inclusion de Kamëz et de son vote majoritairement à droite dans la municipalité de Tirana aurait clairement mis en danger la majorité de gauche traditionnellement portée au pouvoir dans la capitale. En forçant le trait, on peut dire que Tirana, miroir des régionalismes, fragmente ces derniers dans ses territoires institutionnels, peine à les mêler dans son espace social et à les assumer dans sa manière de se projeter. Seul le champ symbolique semble investi : dernier en date des projets successifs de rénovation urbaine du centre de Tirana, la nouvelle place Skanderbeg, rénovée en 2017, prend la forme désormais d'un immense carré central légèrement incliné constitué de dalles de pierres naturelles venues de toutes les régions albanaises (y compris celles situées en Macédoine et au Kosovo), avec l'idée que «la place est le lieu qui transforme un groupe d'individus en une communauté $»^{25}$.

L'espace urbain post-socialiste albanais a été le théâtre d'une transition tumultueuse, véritable renversement suivant 30 années de développement urbain maîtrisé et décentralisé. A la chute du régime, le réseau des villes petites et moyennes a tenu dans un premier temps, au prix d'une ruralisation importante, alors que les grandes villes développaient un caractère dual lié à des séquences migratoires contrastées. Après une 
première décennie de boom du bâti informel, le redéploiement de l'économie de la construction dans le Grand Tirana, sous forme d'une suburbanisation dense, est venu confirmer la ségrégation dessinée par le tissu informel, renforcé en cela par une réforme institutionnelle excluant les banlieues d'immigration du périmètre municipal. La formidable croissance de l'aire métropolitaine Tirana-Durrës a permis de remédier à la sous-urbanisation, mais ce "miroir » urbain de l'Albanie présente un visage plus fragmenté que jamais.

\section{BIBLIOGRAPHIE}

ALIAJ B. (2003), Albania: A short History of Housing and Urban Development Models during 1945-1990, Tirana.

ALIAJ B., LULO K. \& MYFTIU G. (2003), Tirana: The Challenge of Urban Development, Tirana, Cetis. BERTAUD A. (2006), Urban development in Albania: the success story of the informal sector, http:// alainbertaud.com/wp-content/uploads/2013/06/AB_Albania-Urban-informal-Sector-paper.pdf. BIBERAJ E. (1990), Albania. A Socialist Maverick, Boulder, Westview Press.

ÇARO E. (2013), « Winners or Losers? The Adjustment Strategies of Rural-to-Urban Migrants in Tirana, Albania », Journal of Ethnic and Migration Studies, 39, 3, pp. 501-519.

COUDROY DE LILLE L. (2009), « Relire la ville socialiste », Histoire urbaine, Société française d'histoire urbaine (SFHU), pp. 5-13.

COUDROY DE LILLE L. (2016), « Introduction - Entre Est et Ouest : une européanisation urbaine », Revue Géographique de l'Est, 56, 3-4.

DANEMARK B. (1993), « Post-war urban and regional development in Albania », Habitat international, 17, 2, pp. 73-90.

DANIEL O. (1989), « L'Albanie nouvelle : le développement d'une centralité et les problèmes de la politique régionale ", Régions et pouvoirs régionaux en Europe de l'Est et en URSS, Paris, Masson, pp. 21-26.

DANIEL O. (1996), « L’Albanie libérée des légendes », Géographie Universelle. Europes orientales, Russie, Asie centrale, Paris, Belin-Reclus.

DE RAPPER G. (2004), « Les Guègues et les Tosques existent-ils ? L'opposition Nord/Sud en Albanie et ses interprétations ", Espaces, Population, Sociétés, 3, pp. 625-640.

FRENCH R.A., HAMILTON I. (1979), Socialist City: Spatial Structure and Urban Policy, New York, John Wiley \& Sons Ltd.

HIRT S. (2013), « Whatever Happened to the (Post)socialist City? », Cities, 32, 1, p. 29.

JANDOT G. (1981), Hiérarchie urbaine en R.P.S. d'Albanie. Une image spatiale de l'édification du socialisme?, Université Paul Valéry, Montpellier. 
JARNE A., SCHULER M. (2017), « Recompositions territoriales dans l'Albanie postcommuniste : la métropole au détriment des régions? ", La population des Balkans à l'aube du XXI ${ }^{\text {ème }}$ siècle, Skopje, pp. 355-370.

LELAJ O. (2013), «'From peasants to workers': An outline of the proletarisation process during state-socialism in Albania ", Social Practices and Local Configurations in the Balkans, Tirana, UET Press, pp. 41-64.

LERCH M. (2013), « Urbanisation et transition de la fécondité en Albanie », Revue Quetelet, 1, 1, pp. 73-93.

LERCH M. (2014), «The Role of Migration in the Urban Transition: A Demonstration from Albania », Springer, Demography.

MURRAY P., SZELENYI I. \& MARTINET F. (1988), « L'anti-urbanisme des États socialistes », Les Annales de la recherche urbaine, 38, pp. 23-30.

PETROVIC M. (2005), Cities after socialism as a research issue, Discussion papers (South-East Europe series), DP34, Centre for the Study of Global Governance, London School of Economics and Political Science, London.

POJANI D. (2010), « Urbanization of Post-communist Albania: Economic, Social and Environmental Challenges », Debatte: Journal of Contemporary Central and Easter Europe, 17, 1, pp. 8597.

PRATO G. (2017), « Views of Migrants and Foreign Residents: A Comparative European Perspective », Migration of Rich Immigrants. Gender, Ethnicity and Class, Palgrave, pp. 179-198.

SHAMETI E., DHULI E., JAHJA T., JARNE A., SCHULER M. \& VOKOPOLA Z. (2014), Albania Communes and Municipalities Typology, Tirana, Republic of Albania Institute of Statistics (Instat).

SINTÈS P. (2010), La raison du mouvement. Territoires et réseaux de migrants albanais en Grèce, Paris, L'atelier méditerranéen.

SJÖBERG Ö. (1994), « Rural Retention in Albania: Administrative Restrictions on Urban-bound Migration », East European Quarterly.

TOSICS I. (2003), « City Development in Central and Eastern Europe since 1990: The impact of Internal and External Factors », Globalisation and Transformation in Eastern and Central European Cities, New York, The United Nations University.

\section{NOTES}

1. Par opposition à une école dite "écologique » qui considère simplement un retard de développement des villes socialistes.

2. L'urbanisation se fait cependant avec un certain retard sur l'industrialisation, les villes ne parvenant pas à absorber la main-d'œuvre qu'elles attirent. C'est le phénomène de la «sousurbanisation » tel que décrit par Murray et Szelenyi (1988). Voir à ce propos chez Lelaj (2013) le cas de la ville minière de Bulqizë.

3. La population des localités (villages et villes) depuis 1945 est connue grâce à la saisie effectuée par le géographe A. Bërxholli au sein d'un corpus à publier. La définition de la population urbaine et son décompte sur la durée en Albanie se heurte aux problèmes suivants : 1) mutations des niveaux institutionnels ; 2) à certaines périodes, intégration de population rurale dans des unités urbaines (une municipalité urbaine peut contenir, outre sa ville, des villages, unités rurales) ; 3) pour la période récente, inadéquation entre les définitions administratives et statistiques de 
l'urbain et la réalité de la suburbanisation ; 4) ajout de nombreuses unités urbaines durant la période communiste (« villes nouvelles ») ; 5) difficulté d'accès aux données.

4. Voir en particulier Sjöberg (1994).

5. Dès 1960, rupture avec l'URSS, puis après 1980, rupture avec la Chine, isolement diplomatique et économique.

6. En témoigne, par exemple, la réticence et les stratégies développées pour se soustraire au principe de la « rotation des cadres » ou conserver un appartement à Tirana (par exemple en divorçant), ou encore l'hypothèse d'une « diverted migration » décrite par Sjöberg, c'est-à-dire que l'immigration n'étant pas possible vers Tirana, elle se détourne vers les coopératives agricoles situés à ses environs immédiats, qui soulignent l'attractivité propre à Tirana.

7. Le recensement de 2001 donne les lieux de résidence à la naissance et en 1989. Manquent les personnes décédées dans l'intervalle et celles qui ont émigré.

8. La seconde partie de la typologie des communes de l'Instat (Shameti et al., 2014) propose une cartographie très fine des agglomérations urbaines albanaises qui illustre bien ces phénomènes et les ruptures marquées entre les centres et leurs nouvelles banlieues.

9. L'émigration entre 1989 et 2001 est estimée localement en comparant la somme de population qui a déclaré en 2001 résider dans une commune en 1989 à celle qui avait été effectivement recensée dans cette commune en 1989. La différence entre les deux est la somme de la mortalité et de l'émigration. On affecte ensuite de manière uniforme $60 \%$ de cette somme à l'émigration, $40 \%$ à la mortalité. Les ordres de grandeur sont corrects (env. 500000 départs et 0,6 \%o de mortalité annuelle, mais il est clair qu'il s'agit là d'une estimation très grossière).

10. Jusqu'en 2015, l'Albanie comptait 373 communes (rurales) et municipalités (urbaines). À cette date, une importante réforme territoriale-administrative a ramené ce nombre à 61 municipalités. Ces calculs se basent sur l'ancien découpage.

11. La ligne jaune est la limite à l'extension de Tirana posée durant le communisme, elle est demeurée la limite de la municipalité jusqu'en 2015, excluant les importantes banlieues informelles des années 90.

12. Plusieurs travaux, cités par Coudroy de Lille (2016, p. 6), soulignent l'inachèvement de la métropolisation à l'Est. Le phénomène était criant pour l'Albanie communiste. Depuis 2000, Tirana rattrape son retard, de manière spectaculaire pour ce qui concerne la concentration des biens et équipements au niveau national, mais également par son intégration dans les réseaux européens et mondiaux. Elle est, par exemple, depuis 2016 classée " gamma » et donc considérée « world city » par le GaWC (http://www.lboro.ac.uk/gawc/gawcworlds.html).

13. Voir De Rapper (2004), pour la pertinence de cette interprétation, et Daniel (1996) ou Biberaj (1990) sur certains aspects du contenu de cette opposition.

14. Voir la démonstration de Danemark B. (1993).

15. Voir par exemple Sintès P. (2010).

16. Voir l'atlas urbain produit par l'Instat à des niveaux géographiques très fins sur la base du recensement de 2011 (Shameti et al. 2014).

17. Les données de 2011 ne nous ont pas été accessibles avec une finesse spatiale suffisante. Elles sont par contre visibles au niveau des communes dans le tableau 3.

18. Quartier réservé à l'élite du régime durant le communisme.

19. Les mesures de densité qui suivent sont essentiellement tirées du recensement de 2001 et calculées au niveau des secteurs de recensements de Tirana (définition d'avant 2015, à l'intérieur de la « ligne jaune »), desquels on a retranché les zones industrielles, les secteurs administratifs, les parcs, etc. Ces densités ont été mesurées sur la base de l'âge des immeubles et de la population enregistrée dans chacun.

20. Bertaud (2006) distingue 3 phases, celle strictement informelle, puis l'émergence d'un secteur formel et la consolidation du secteur informel, enfin la consolidation du secteur formel et la régularisation du secteur informel, depuis 2005 environ. 
21. Plan directeur de 1989, voir Aliaj et al. (2003, p. 63).

22. Comme les toutes nouvelles «Rolling Hills Luxury Residences » qui se prévalent d'être les premières dans leur genre en Albanie, les plus luxueuses, qui ont élu domicile à côté d'un mall haut de gamme, le « Tirana East Gate » et d'une université privée.

23. De manière systématique, les banlieues d'immigration ont été exclues, celles de la suburbanisation récentes incluses.

24. Ce que relevait en 2016 sans citer de cas précis le rapporteur du Conseil de l'Europe (http:// www.balkaninsight.com/en/article/albanian-territorial-reform-slow-and-confusing-03-23-2016). 25. Discours de l'ancien maire de Tirana et actuel Premier ministre Edi Rama lors de l'inauguration.

\section{RÉSUMÉS}

Après 30 années d'un développement urbain maîtrisé et décentralisé, l'espace urbain albanais a été le théâtre d'une transition brutale. A la chute du régime en 1991, le réseau des villes petites et moyennes mis en place durant le communisme a tenu dans un premier temps, au prix d'une importante «ruralisation » des espaces urbains, alors que les grandes villes développaient un caractère dual, opposant centres et nouveaux quartiers informels aux compositions sociodémographiques bien différentes, en raison de séquences migratoires contrastées. Après une première décennie marquée par le boom des constructions informelles, le redéploiement du secteur de l'immobilier dans le Grand Tirana a produit une suburbanisation dense, qui est venue confirmer la ségrégation dessinée par le tissu informel, renforcée en cela par une réforme institutionnelle excluant les banlieues d'immigration du périmètre municipal. La formidable croissance de l'aire métropolitaine Tirana-Durrës a permis de remédier à la sous-urbanisation, mais ce miroir urbain de l'Albanie présente un visage plus fragmenté que jamais.

After 30 years of controlled and decentralized urban development, the Albanian urban space has gone through a brutal transition. At the fall of the regime in 1991, the network of small and medium-sized cities set up during communism initially survived, at the cost of an important "ruralization" of urban spaces, while the big cities developed a dual character, opposing centers and new "informal" neighborhoods with very different socio-demographic compositions, due to contrasting migratory sequences. After a first decade of informal boom, the redeployment of the real estate market in Greater Tirana, in the form of a dense suburbanization, has confirmed the segregation drawn by the informal urban fabric, further reinforced by an institutional reform excluding immigration suburbs of the municipal territory. The tremendous growth of the TiranaDurrës metropolitan area has allowed addressing the under-urbanization problem, but this urban mirror of Albania seems more fragmented than ever.

\section{INDEX}

Keywords : Albania, postsocialist, Tirana, urbanization, migration, segregation, suburbanization Mots-clés : Albanie, postsocialisme, Tirana, urbanisation, migration, ségrégation, suburbanisation 
AUTEUR

ALAIN JARNE

Doctorant au Laboratoire des SIG (LASIG), Ecole polytechnique fédérale de Lausanne (EPFL), alain.jarne@epfl.ch 